\title{
Top Transmission Grating GaN LED Simulations for Light Extraction Improvement
}

\author{
Simeon Trieu ${ }^{\mathrm{a}, \mathrm{b}}$, Xiaomin Jin ${ }^{*}$, b, Ashton Ellaboudy ${ }^{\mathrm{a}}$, Bei Zhang ${ }^{\mathrm{b}}$, Xiang-Ning Kang ${ }^{\mathrm{b}}$, Guo-Yi \\ Zhang ${ }^{\mathrm{b}}$ Xiong Chang ${ }^{\mathrm{b}}$, Wei Weib ${ }^{\mathrm{b}}$, Sun Yong Jian ${ }^{\mathrm{b}}$, and Fu Xing Xing ${ }^{\mathrm{b}}$ \\ ${ }^{a}$ Electrical Engineering Department, 1 Grand Avenue, \\ California Polytechnic State University, San Luis Obispo, CA, USA, 93407-9000; \\ ${ }^{\mathrm{b}} \mathrm{School}$ of Physics and State Key Laboratory for Artificial Microstructures and Mesoscopic \\ Physics, Peking University, Beijing, China, 100871
}

\begin{abstract}
We study the top transmission grating's improvement on GaN LED light extraction efficiency. We use the finite difference time domain (FDTD) method, a computational electromagnetic solution to Maxwell's equations, to measure light extraction efficiency improvements of the various grating structures. Also, since FDTD can freely define materials for any layer or shape, we choose three particular materials to represent our transmission grating: 1) nonlossy p-GaN, 2) lossy indium tin oxide (ITO), and 3) non-lossy ITO $(\alpha=0)$. We define a regular spacing between unit cells in a crystal lattice arrangement by employing the following three parameters: grating cell period (A), grating cell height (d), and grating cell width (w). The conical grating model and the cylindrical grating model are studied. We also presented in the paper directly comparison with reflection grating results. Both studies show that the top grating has better performance, improving light extraction efficiency by $165 \%$, compared to that of the bottom reflection grating $(112 \%)$, and top-bottom grating $(42 \%)$. We also find that when grating cells closely pack together, a transmission grating maximizes light extraction efficiency. This points our research towards a more closely packed structure, such as a 3-fold symmetric photonic crystal structure with triangular symmetry and also smaller feature sizes in the nano-scale, such as the wavelength of light at $460 \mathrm{~nm}$, half-wavelengths, quarter wavelengths, etc.
\end{abstract}

Keywords: Gallium Nitride, Light-Emitting-Diode, grating

\section{INTRODUCTION}

Light emitting diodes represent an expanding field of research to develop brighter, cheaper, and more energy efficient light sources. Fiber optic systems, light bulbs, display technology, and machine vision all make use of LEDs as either the ideal light source or low cost alternative to laser diodes. In order to be a viable and cost-effective solution, LEDs must meet the industry's stringent performance criteria: minimize power consumption, generate minimal heat, and maximize extracted lumens per watt. Currently, two LED optimization methods improve these performance characteristics: increasing electroluminescent efficiency and increasing light extraction efficiency [1]. Electroluminescent efficiency measures how efficiently electrons in a direct bandgap material are converted into photons of light, while light extraction efficiency measures how efficiently those generated photons are extracted from the LED without absorption or reflection. This paper's research seeks to increase LED light extraction efficiency of Gallium Nitride (GaN) LEDs. The three key inefficiencies in light extraction efficiency of GaN LEDs are: 1) GaN's high refractive index, and therefore low critical angle at the semiconductor-air interface, 2) the absorption of light within the device at the multiple quantum well (MQW) region, as well as dislocations and defects in the GaN crystal, and 3) inefficient device geometry [2-4].

*xjin@calpoly.edu; phone 1 805-756-7046; fax 1 805-756-1458; www.ee.calpoly.edu

Physics and Simulation of Optoelectronic Devices XIX, edited by Bernd Witzigmann,

Fritz Henneberger, Yasuhiko Arakawa, Alexandre Freundlich, Proc. of SPIE Vol. 7933,

79331Y · @ 2011 SPIE · CCC code: 0277-786X/11/\$18 · doi: 10.1117/12.871595

Proc. of SPIE Vol. 7933 79331Y-1 
The first key limitation to light extraction efficiency results when a GaN device layer's low critical angle traps generated light internally. In other words, much of the light generated within the devices is totally internally reflected back within the device rather than escaping as emitted light. Fig. 1 shows the typical problem of a GaN LED with such a high refractive index. The light blue photon coming in at a high angle above the critical angle reflects internally, while the dark blue photon coming in at a low angle below the critical transmits to the air. If the critical angle is sufficiently low, then generated photons will find the cone of acceptable escape angles too narrow to produce significant light extraction. Fig. 1(a) shows this narrow cone of acceptance, normal to the semiconductor surface. The cone is traced from the critical angle of incidence for a point at the origin. Any photon that strikes the surface at an angle larger than the critical angle will be reflected internally, thereby decreasing light extraction.

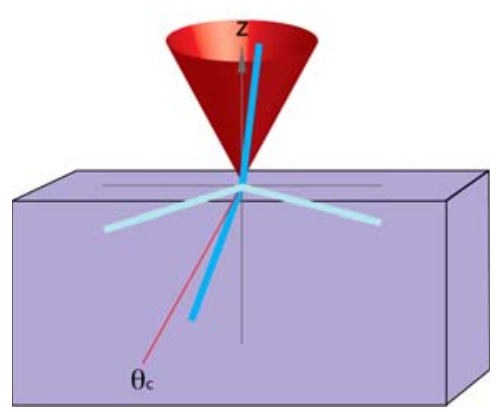

(a)

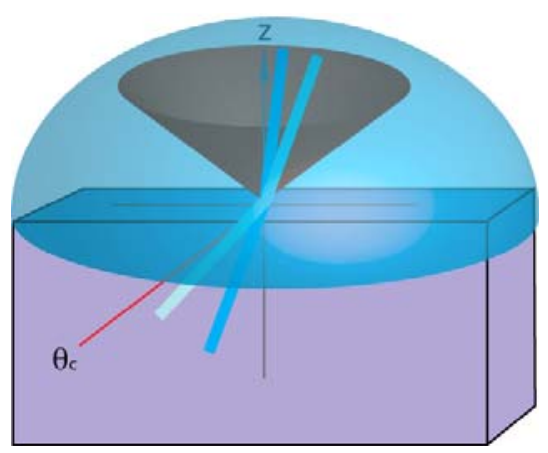

(b)

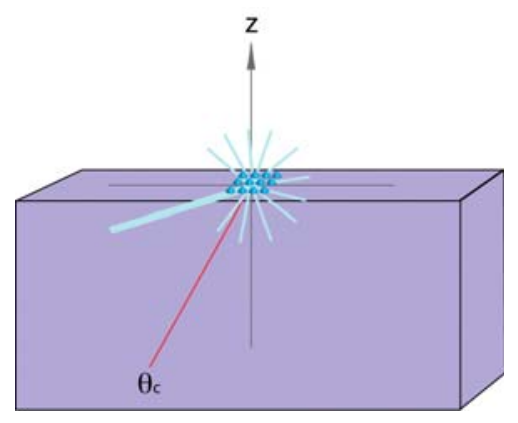

(c)

Fig. 1 - Total Internal Reflection Model: (a) cone of transmitted angles and total internal reflection, (b) LED with enhanced cone of transmitted angles, due to affixed resin dome, and (c) incident beam scattering via Bragg diffraction at the grating interface.

A number of solutions have been proposed to solve this reflection problem. First, a common approach affixes a domeshaped resin over the LED to create a larger escape angle out of the device. Fig. 1 (b) shows how the critical angle might be increased and therefore increase light extraction efficiency. The lower index difference between GaN and the resin enlarges the cone when compared to the model in Fig. 1(a). In addition, the resin's dome shape ensures that the extracted light at the semiconductor-resin interface meets the resin-air interface at a normal or a low enough angle to allow for greater light extraction [5]. To further mitigate the problems of total internal reflection, the emission surface can be patterned to form a transmission grating that offers the light more angles of escape, as shown in Fig. 1(c). A transmission grating allows partial transmission at angles above the critical angle. Instead of light reflecting at grating surfaces, the light scatters at these surfaces for partial transmission of otherwise completely reflected light. A variety of methods exist to construct these gratings, including wet etching with an amorphous sacrificial layer [6-9] or laser etching to obtain a more periodic spacing [10-14]. Any of these methods can etch a transmission grating on a semiconductor surface, each with their respective advantages and disadvantages. In previous literature, patterning of an $\mathrm{Ag}$ reflector plate in either a pillar or hole grating shape can form a reflection grating [15-16]. 


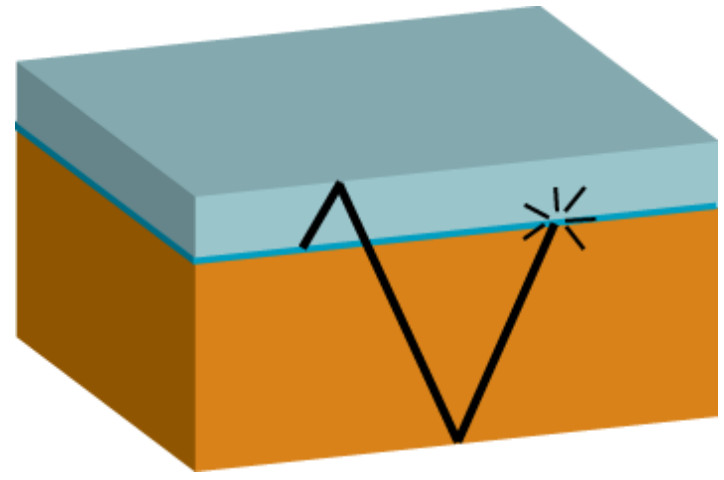

Fig. 2 - Absorption within the LED.

A light emissive device's second key limitation results from the absorption of generated light within the device at the multiple quantum well region or at defects within the GaN crystal structure, as shown in Fig. 2. The top layer is a p$\mathrm{GaN}$ semiconductor, followed by a MQW active layer, and an $\mathrm{n}-\mathrm{GaN}$ semiconductor. The photon that cannot escape, due to an incident angle higher than the critical angle, travels within the LED and eventually absorbs into the MQW active layer by exciton or phonon-assisted interactions. Photon absorption constitutes a critical aspect of not only light extraction efficiency, but also of device operation. Since the photon does not escape from the device, it does not contribute to light output and also generates heat that is dispersed throughout the diode by a phonon interaction or lattice vibration. Increased heat within LED luminaries seriously degrades LED lifetime, alters the emitted wavelength of light, and consumes energy that could be used for light emission. The MQW region is the source of emitted light within the diode and deserves particular attention. A generated photon emitted from the MQW region also matches the band gap energy of that same layer, and therefore, the MQW can readily absorb generated photons and does so at a high rate. Fig. 2 illustrates how every pass across the MQW layer increases the chances of absorption. The larger the MQW layer is, the greater the chances of absorption. Therefore, there is a trade-off in terms of light generation and photon absorption when designing layered MQW structures in LEDs. Photons must be extracted, both in greater quantity and speed, before absorption occurs $[4,17,18]$. A grating structure will solve this issue by creating more angles of escape. Grating structures can be patterned in many shapes including: pyramidal, spherical, conical, cylindrical, and others, but only a few can be fabricated with great success. For example, the modified laser-lift off (M-LLO) technique can pattern holes a 4-micron period instead of etching a random structure. The technique etches the nanostructure onto u-GaN using a sapphire backplane, UV light, and a high power KrF laser. Peking University's (PKU) experiment varied the grating depths from $75 \mathrm{~nm}$ to $120 \mathrm{~nm}$ [19]. Imprint lithography, that can also produce similar air holes that measure $180 \mathrm{~nm}$ in diameter, with a depth of $67 \mathrm{~nm}$, and a period of $295 \mathrm{~nm}$ [20].

The research presented in this paper simulates a variety of top grating types and explores the transmission of light above the critical angle, and uncovers the mechanics behind light extraction from grating structures. Gratings can vary in following ways: (1) by placing the grating on different layers within the thin-film LED, (2) by varying the density of grating cells on the grating layer, (3) by altering the shape of the grating cells, and (4) by increasing the symmetry of the photonic crystal grating. This paper focuses on the transmission grating structure, a single grating structure that approximately doubles the light extraction from an LED.

\section{SIMULATION MODEL}

Our previous publication [15] presented the bottom reflection grating design. Here we consider the top transmission grating's effects on GaN LED light extraction efficiency, as the structure applies easily to fabrication, while reflection gratings present some difficulties in processing with the M-LLO procedure [15]. We form the same grating cell shapes, cylindrical and conical shaped grating cells, as the reflection grating study shown in Fig. 3. For this LED simulation, we have employed the 2-D the finite difference time domain (FDTD) method, as in the reflection grating studies [15]. The FDTD method offers many significant advantages for grating simulation, including a full-wave 
solution, finely controlled accuracy via the size of the simulation grid and steps, and accounting of all optical phenomenons of reflection, refraction, and diffraction.

The transmission grating structure differs from the reflection grating structure, in that grating cells form pillars instead of holes. Also, since FDTDcan freely define materials for any layer or shape, we choose three particular materials to represent our transmission grating: 1) non-lossy p-GaN, 2) lossy indium tin oxide (ITO), and 3) non-lossy ITO ( $\alpha=0)$. We define a regular spacing between unit cells in a crystal lattice arrangement by employing the following three parameters in Fig. 4, similar to the single reflection grating studies: grating cell period (A), grating cell height (d), and grating cell width (w), which shows the conical grating model in Fig. 4(a) and the cylindrical grating model in Fig. 4(b).

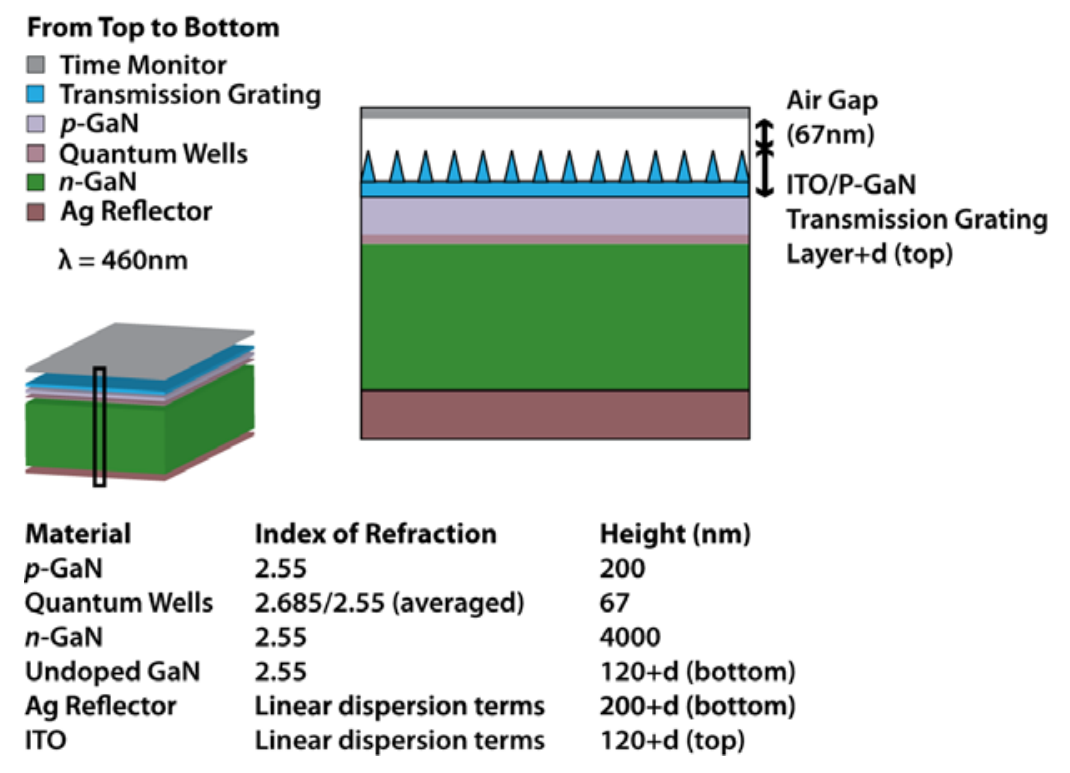

Fig. 3 - Transmission grating GaN LED model.

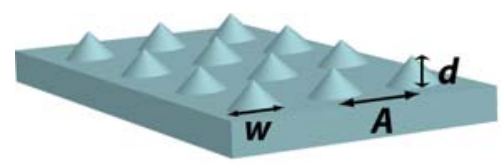

(a)

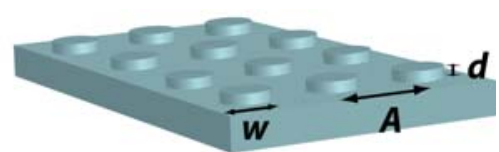

(b)

Fig. 4 - Transmission Grating Types: (a) conical and (b) cylindrical.

\section{SIMULATION RESULTS AND DISSCUSIONS}

We obtain the results presented in Fig. 4 to Fig. 7 by sweeping through the grating parameters, exactly like the reflection grating studies [15]. The grating parameters (ie. A, w, and d) are combined in all possible combinations in the following specified parameter ranges: $\mathrm{A}=1$ to $6 \mu \mathrm{m}, \mathrm{w}=1$ to $6 \mu \mathrm{m}$, and $\mathrm{d}=50$ to $200 \mathrm{~nm}$. Note that $\mathrm{w}>\mathrm{A}$ is an invalid parameter combination. Each material case analyzes the significant findings in the following sections. 


\subsection{Non-Lossy p-GaN Transmission Grating Simulation Results}

We present the conical and cylindrical p-GaN transmission grating simulation results in Fig. 5 and 6. Conical p-GaN transmission gratings maximize light extraction efficiency in the range of $A=1 \mu \mathrm{m}$ and $\mathrm{A}=2 \mu \mathrm{m}$, as shown in Fig. 5(a) and Fig. 5(b), and reveal both non-lossy $\mathrm{p}-\mathrm{GaN}$ conical transmission gratings maximize average power at $\mathrm{A}=1 \mu \mathrm{m}$, $\mathrm{w}=1 \mu \mathrm{m}, \mathrm{d}=200 \mathrm{~nm}$, which output a maximum light improvement of $165 \%$ over the non-grating case. Specifically, Fig. 7(a) reveals a trend: when $A$ approaches the dimensions of $w$, light extraction efficiency generally increases. The grating appears largely unaffected by grating period or grating height until the transmission grating begins to reach feature sizes comparable to the wavelength of light. Again, this suggests that grating efficiency would be improved in the nano-scale, but also, light output increases with more transmission grating cells fabricated on the surface of an LED.

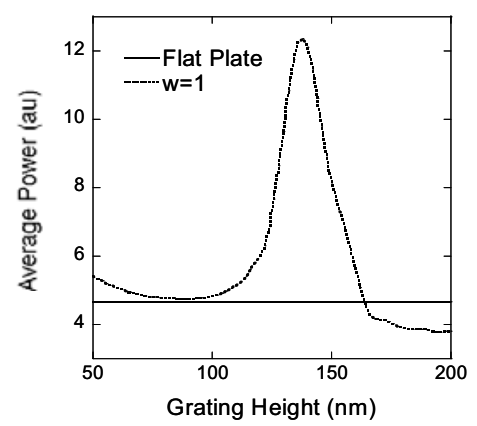

(a)

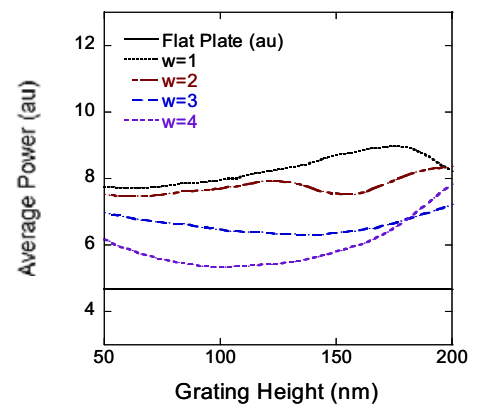

(d)

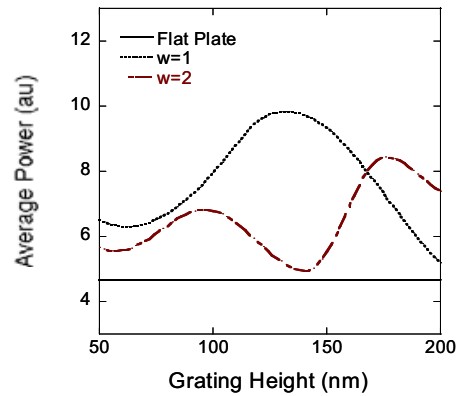

(b)

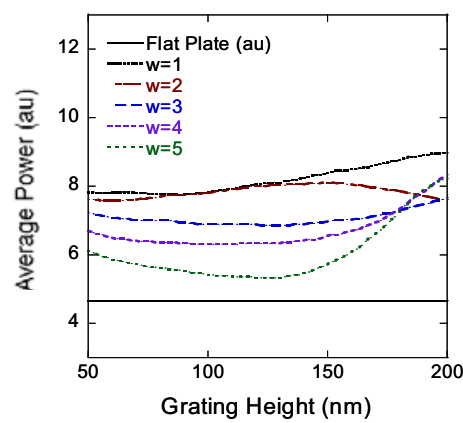

(e)

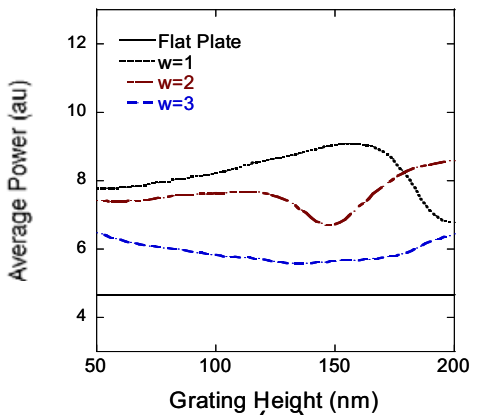

(c)

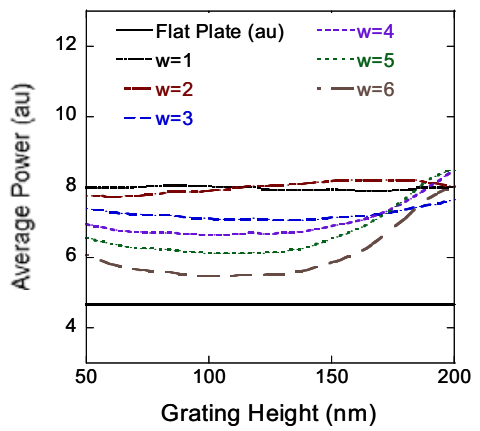

(f)

Fig. 5 - Average power for the conical non-lossy p-GaN transmission grating case: (a) $A=1$, (b) $A=2$, (c) $A=3$, (d) $A=4$, (e) $A=5$, and (f) $A=6 \mu \mathrm{m}$.

For non-lossy, $\mathrm{p}-\mathrm{GaN}$ cylindrical pillar transmission gratings, the $\mathrm{A}=\mathrm{w}$ case continues to produce results above the $\mathrm{A}>\mathrm{w}$ case. Again, due to the transformation of the basic shape of the grating unit cell from cylindrical pillars into 4star shaped holes, an undesirable grating pattern in terms of light extraction improvement. Fig. 6 shows the full simulation set for cylindrical pillars. The peak power vs. grating height graph in Fig. 7(b) shows very clearly that each of the cases where $\mathrm{A}=\mathrm{w}$, decays into the same unfavorable four starred-hole shape, as in $\mathrm{A}=1 \mu \mathrm{m}, \mathrm{w}=1 \mu \mathrm{m}$. Otherwise, the optimal grating strikes a balance between maximizing $w$ with respect to $A$, maximizing the number of transmission grating cells on top of the LED, and maintaining the cylindrical pillar grating structure. The non-lossy p-GaN cylindrical pillar transmission grating case of $\mathrm{A}=2 \mu \mathrm{m}, \mathrm{w}=1 \mu \mathrm{m}$ maximizes light extraction efficiency by balancing all of these conditions. 


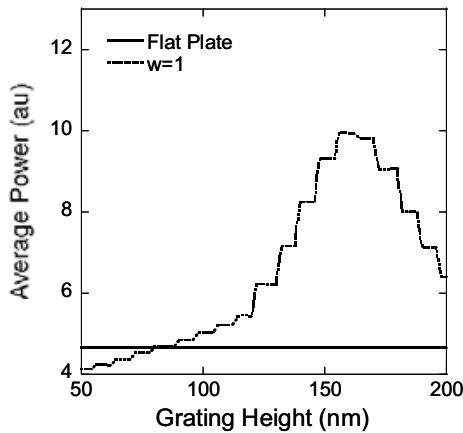

(a)

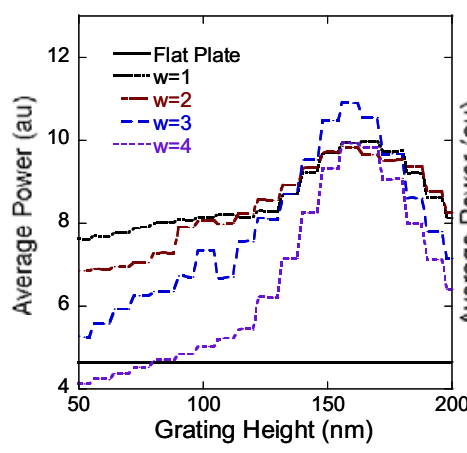

(d)

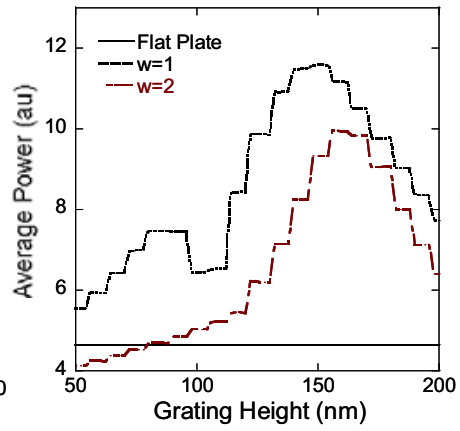

(b)

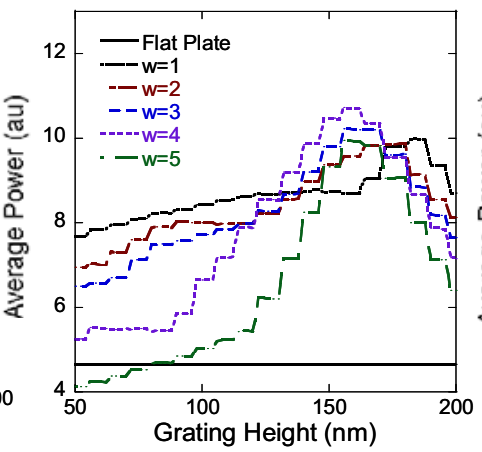

(e)

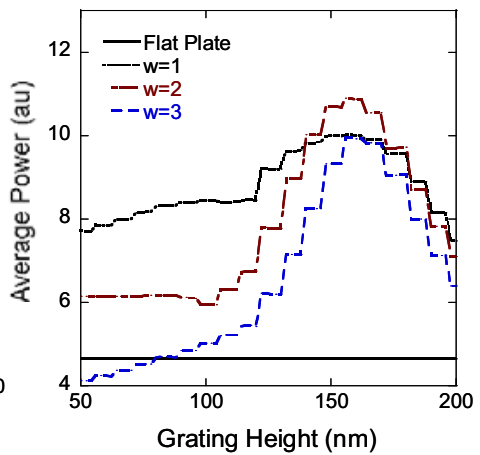

(c)

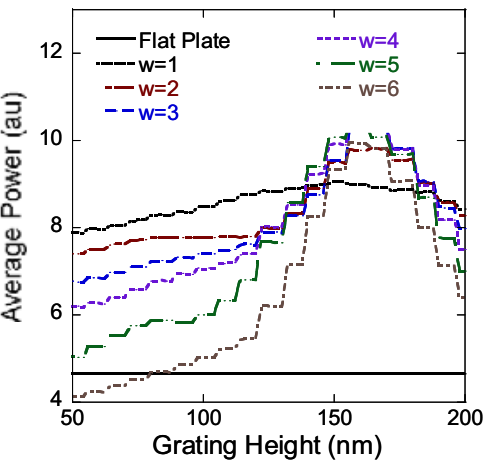

(f)

Fig. 6 - Average power for the cylindrical non-lossy p-GaN transmission grating case: (a) A=1, (b) A=2, (c) A=3, (d) A=4, (e) $\mathrm{A}=5$, and (f) $\mathrm{A}=6 \mu \mathrm{m}$.

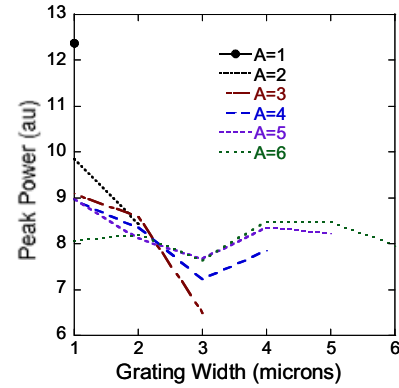

(a)

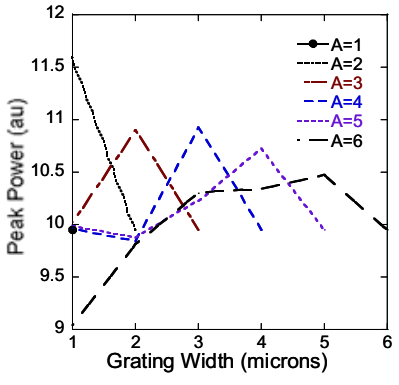

(b)

Fig. 7 - Peak Power vs. Grating Width for (a) conical and (b) cylindrical non-lossy p-GaN transmission gratings.

\subsection{Lossy ITO Transmission Grating Simulation Results}

Next, we simulated a lossy ITO transmission grating structure. Fig. 8 shows the full simulation set for the lossy conical ITO transmission grating. A grating period of 4,5 , and $6 \mu \mathrm{m}$ maximizes light extraction efficiency at approximately $8.4 \mathrm{au}$. Again, the peak power maximizes when $w$ equals $A$. The more grating cells that pack closely together, the greater the light extraction efficiency, maximizing at $A=w$. All transmission gratings in this simulation set improve light extraction efficiency. With the lossy conical ITO transmission grating, light extraction efficiency maximizes at about $80 \%$. At the very minimum, the lossy conical ITO transmission grating results in about a $30 \%$ increase in light extraction efficiency. Also, Fig. 9 shows the full simulation set for the lossy cylindrical ITO transmission grating. A 
grating height of $118 \mathrm{~nm}$ at $A=1 \mu \mathrm{m}$ and $w=1 \mu \mathrm{m}$, maximizes light extraction efficiency, resulting in a light extraction increase of $95 \%$ over the non-grating case.

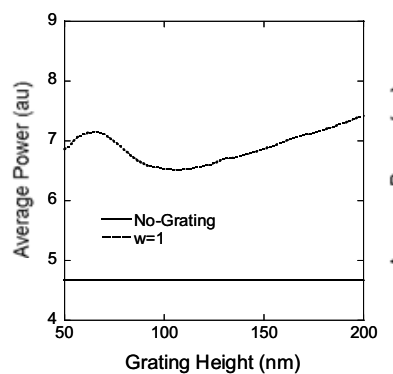

(a)

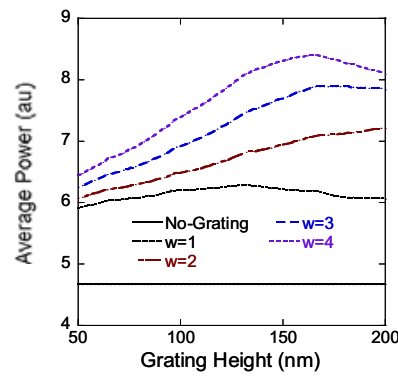

(d)

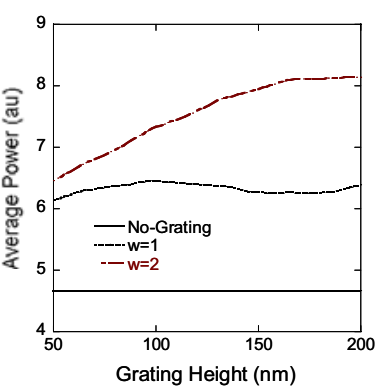

(b)

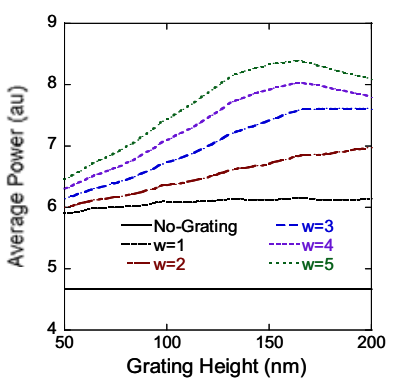

(e)

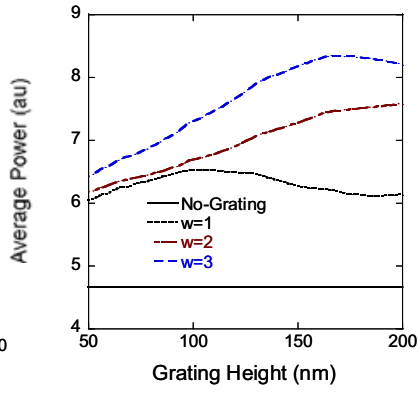

(c)

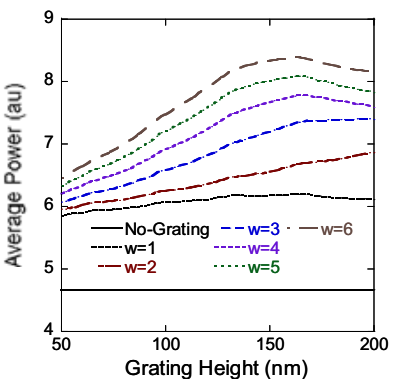

(f)

Fig. 8 - Average power for the lossy conical ITO transmission grating case: (a) $A=1$, (b) $A=2$, (c) $A=3$, (d) $A=4$, (e) $A=5$, and (f) $\mathrm{A}=6 \mu \mathrm{m}$.

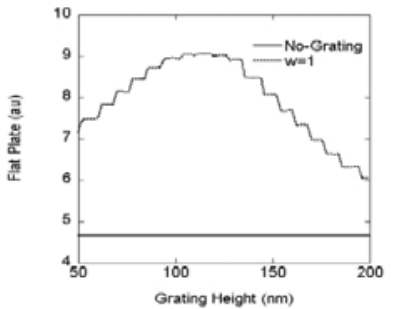

(a)

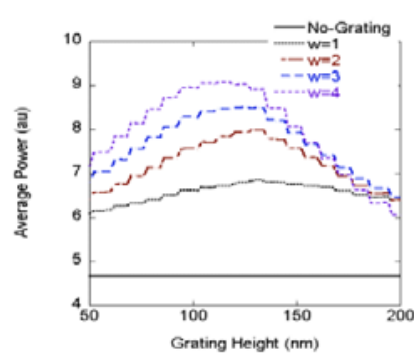

(d)

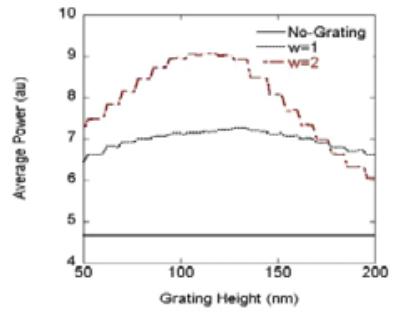

(b)

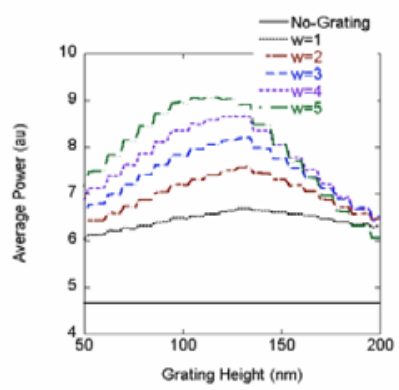

(e)

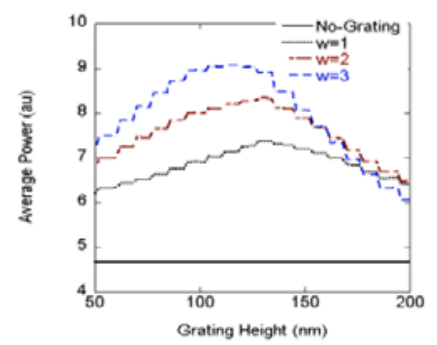

(c)

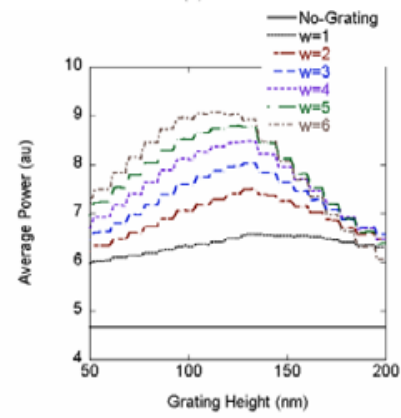

(f)

Fig. 9 - Average power for the lossy cylindrical ITO transmission grating case: (a) A=1, (b) A=2, (c) A=3, (d) $A=4$, (e) A=5, and (f) $A=6 \mu \mathrm{m}$. 


\subsection{Non-Lossy ITO Transmission Grating Simulation Results}

Finally, we simulated a non-lossy ITO transmission grating structure. Fig. 10 shows the full simulation set for nonlossy ITO conical pillar transmission gratings. The trend closely matches the lossy ITO structure but without the loss factors, resulting in a constant relative drop in output power between the two models. From Fig. 10 and 12(a), the structure optimizes at $\mathrm{A}=4 \mu \mathrm{m}, \mathrm{w}=4 \mu \mathrm{m}, \mathrm{d}=166 \mathrm{~nm}$ with a $90 \%$ improvement. For the non-lossy ITO cylindrical pillar transmission grating, the results show similarities to the lossy ITO structure. From Fig. 4.9 and 4.10(b), the structure optimizes when $A=w$ and d=126 nm, with an improvement of $109 \%$ for all cases where $A=w$.

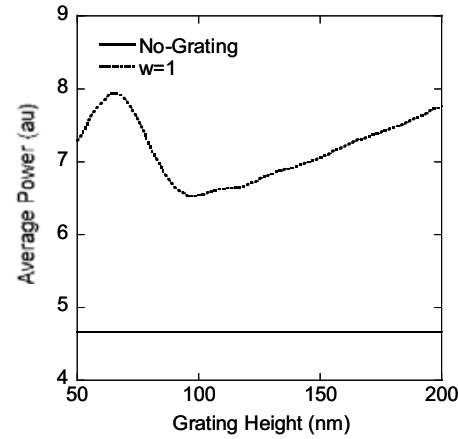

(a)

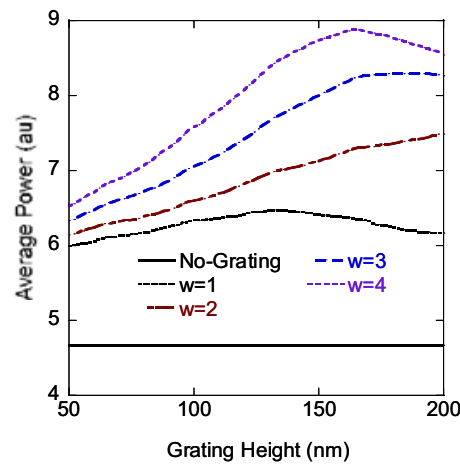

(d)

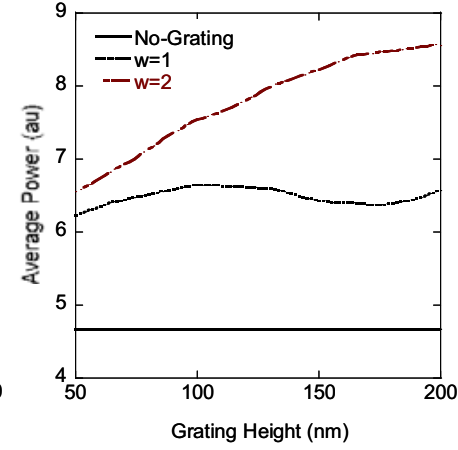

(b)

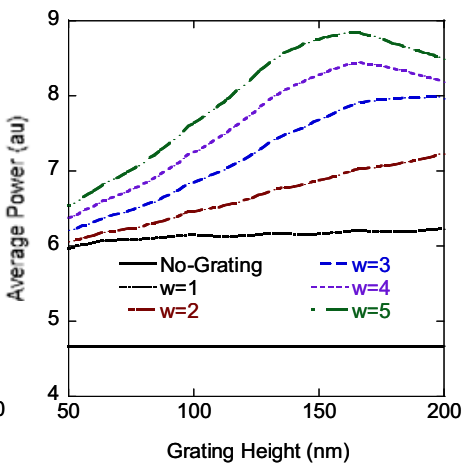

(e)

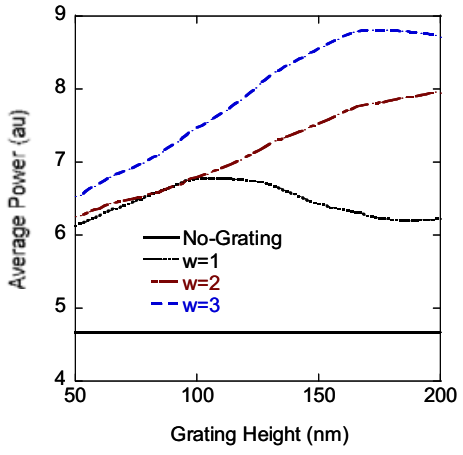

(c)

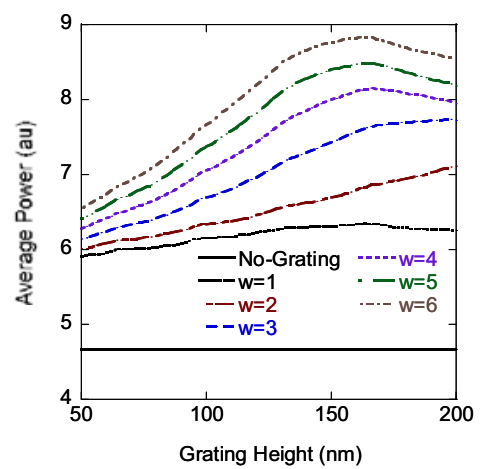

(f)

Fig. 10 Average power for the non-lossy conical ITO transmission grating case: (a) $A=1$, (b) $A=2$, (c) $A=3$, (d) $A=4$, (e) $A=5$, and (f) $\mathrm{A}=6 \mu \mathrm{m}$. 


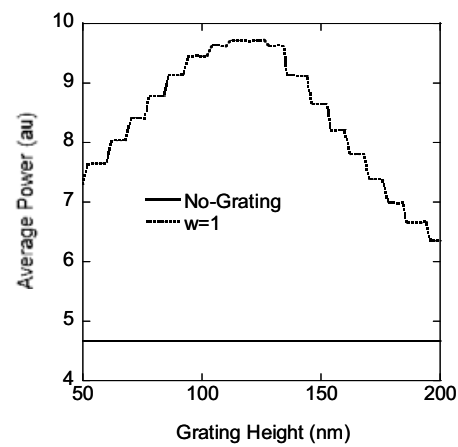

(a)

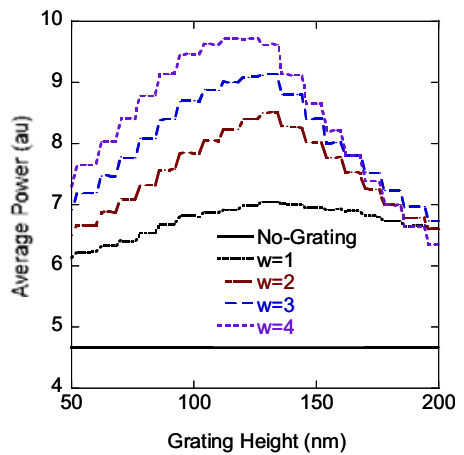

(d)

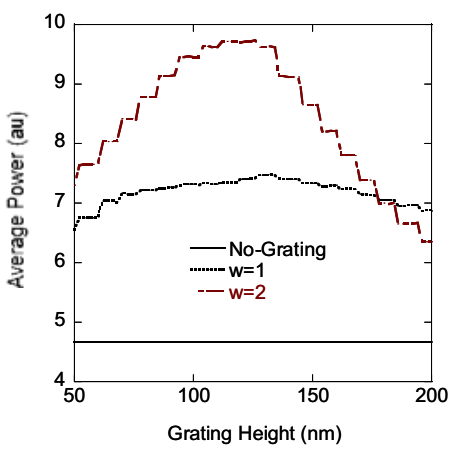

(b)

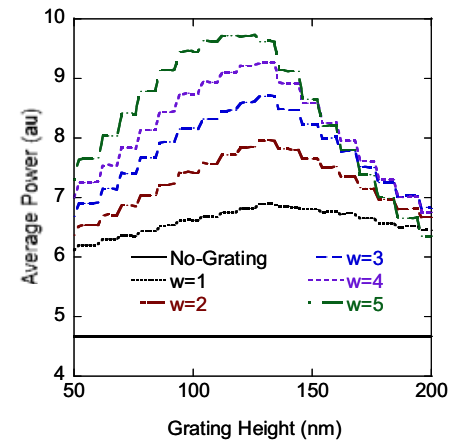

(e)

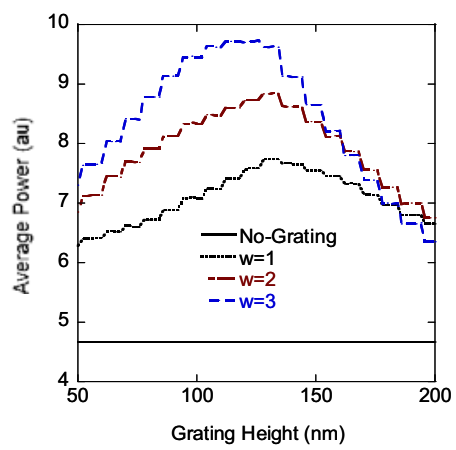

(c)

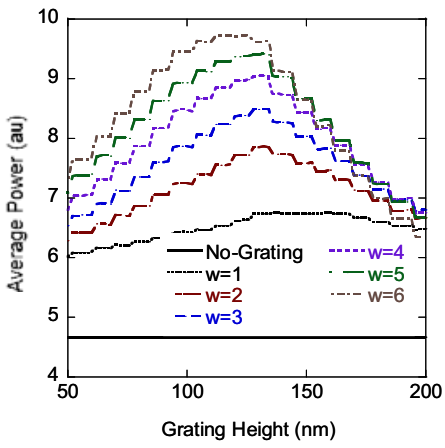

(f)

Fig. 11 Average power for the non-lossy cylindrical ITO transmission grating case: (a) $A=1$, (b) $A=2$, (c) $A=3$, (d) $A=4$, (e) $\mathrm{A}=5$, and (f) $\mathrm{A}=6 \mu \mathrm{m}$.

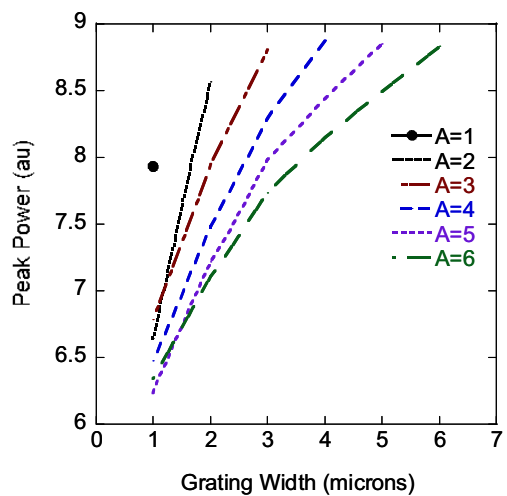

(a)

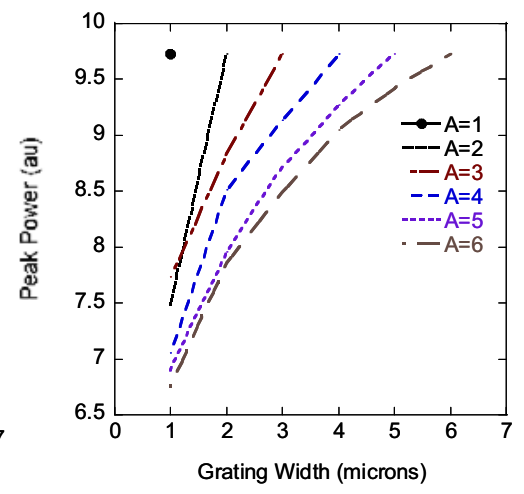

(b)

Fig. 12 Peak power vs. grating width for non-lossy ITO transmission gratings: (a) conical and (b) cylindrical. 


\section{CONCLUSION}

Single transmission grating were simulated using the 2-D FDTD method. Three material types were simulated: nonlossy p-GaN, non-lossy ITO, and lossy ITO. In addition, a conical and cylindrical grating cell shape was simulated for each type of material. The results show that light extraction efficiency was maximized when using a non-lossy conicalshaped $\mathrm{p}-\mathrm{GaN}$ material for a transmission grating. The grating parameters for that particular grating were $\mathrm{A}=1, \mathrm{w}=1$, $\mathrm{d}=138 \mathrm{~nm}$. Regardless of what grating type was used, light extraction efficiency improved by at least $30 \%$ over the reference case (no grating). This research has found what was already agreed in the literature, that grating structures influence light extraction efficiency in the following way: as A compares similarly in dimension to w, light extraction efficiency improves. In other words, when grating cells closely pack, maximum light extraction efficiency should occur. This points our research towards a more closely packed structure, such as a 3-fold symmetric photonic crystal structure with triangular symmetry and also smaller feature sizes in the nano-scale, such as the wavelength of light at $460 \mathrm{~nm}$, half-wavelengths, quarter wavelengths, etc. We also presented in the paper directly comparison with reflection grating results [21]. The studies show that the top grating has better performance, improving light extraction efficiency by $165 \%$, compared to that of the bottom reflection grating $(112 \%)$, and top-bottom grating (42\%). In summary, the top transmission gratings are easy to be fabricated and have best light extraction efficiency compared to the bottom and top/bottom grating structures.

\section{ACKNOWLEDGEMENT}

This project is supported by 1) NSF Grant IRES Award \#1029135 from year 2010 to 2013, 3) Department of the Navy, Office of Naval Research, under Award \# ONR 6-N00014-07-1-1152 in 2008, Award \# ONR 7N000140811209 in 2009, USA, and 3) Chinese National Key Research Lab Collaboration Grant 2010, Peking University in China.

\section{REFERENCES}

[1] Svensk, P. T. Torma, S. Suihkonen, M. Ali, H. Lipsanen, M. Sopanen, A. Odnoblyudov, V. E. Bougrov, "Enhanced electroluminescence in $405 \mathrm{~nm}$ InGaN/GaN LEDs by optimized electron blocking layer,” J. of Crystal Growth, 310, 5154-5157 (2008).

[2] R. H. Horng, C. C. Yang, J. Y. Wu, S. H. Huang, C. E. Lee, D. S. Wuu, "GaN-based light-emitting diodes with indium tin oxide texturing window layers using natural lithography,” Appl. Phys. Lett., 86, 221101 (2005).

[3] H.W. Huang, C. C. Kao, J. T. Chu, H. C. Kuo, S. C. Wang, C. C. Yu, "Improved light-output and electrical performance of InGaN-based light-emitting diode by microroughening of the p-GaN surface," IEEE Phot. Tech. Lett., 17, 9383-9385 (2005).

[4] T. X. Lee, C. Y. Lin, S. H. Ma, C. C. Sun, "Analysis of position-dependent light extraction of GaN-based LEDs," Optics Express, 13, 4175-4179 (2005).

[5] G. Hatakoshi, Y. Hattori, S. Saito, N. Shida, and S. Ninoue, "Device Simulator for Designing High-Efficiency Light-Emitting Diodes,” Jpn. J. Appl. Phys., 46(8B), 5419-5425 (2007).

[6] Y. A. Chen, N. Y. Liang, L. H. Laih, W. C. Tsay, M. N. Chang, J. W. Hong, "Improvement of electroluminescence characteristics of porous silicon LED by using amorphous silicon layers," Elec. Lett., 33, 1489-1490 (1997).

[7] H. W. Huang, C. C. Kao, H. C. Chu, S. C. Wang, C. C. Yu, "Improvement of InGaN-GaN Light-Emitting Diode Performance with a Nano-Roughened p-GaN Surface," IEEE Phot. Tech. Lett., 17, 983-985 (2005).

[8] K. Bao, X. N. Kang, B. Zhang, T. Dai, C. Xiong, H. Ji, G. Y. Zhang, Y. Chen, "Improvement of Light Extraction from Patterned Polymer Encapsulated GaN-Based Flip-Chip Light-Emitting Diodes by Imprinting," IEEE Phot. Tech. Lett., 19, 1840-1842 (2007).

[9] H. Y. Ryu, J. K. Kwang, Y. J. Lee, Y. H. Lee, "Enhancement of Light Extraction from Two-Dimensional Photonic Crystal Slab Structures,” IEEE J. on Sel. Top. in Quant. Elec., 8, 231-237 (2002).

[10] T. Kim, A. J. Danner, K. D. Choquette, "Enhancement in external quantum efficiency of blue light-emitting diode by photonic crystal surface grating,” Elec. Lett., 41, 1138-1139 (2005). 
[11] F. F. Ren, M. B. Yu, J. D. Ye, Q. Chen, G. Q. Lo, D. L. Kwong, "Enhanced Vertical Light Extraction from Ultrathin Amorphous Si-Si3N4 Multilayers with Photonic Crystal Patterns," IEEE Phot. Tech. Lett., 21, 91-93 (2009).

[12] T. Dai, B. Zhang, Z.-S. Zhang, D. Liu, X. Wang, K. Bao, X.-N. Kang, J. Xu, D.-P. Yu, X. Zhu, "Surface Light Extraction Mapping from Two-Dimensional Array of 12-Fold Photonic Quasicrystal on Current Injected GaNBased LEDs," Chinese Phys. Lett., 24, 979-982 (2007).

[13] R. J. Yan, Q. K. Wang, "Enhancement of Light Extraction Efficiency in OLED with Periodic Nano-Structure," IEEE Intl. Conf. on Nano/Micro Eng. And Molecular Sys., Jan. 18-26 (2006).

[14] J. Q. Xi, H. Luo, A. J. Pasquale, J. K. Kim, E. F. Schubert, "Enhanced Light Extraction in GaInN Light-Emitting Diode with Pyramid Reflector," IEEE Phot. Tech. Lett., 18, 2347-2349 (2006).

[15] S. Trieu, X. Jin, B. Zhang, T. Dai, K. Bao, X. N. Kang, G. Y. Zhang, "Light Extraction Improvement of GaN-based Light Emitting Diodes using Patterned Undoped GaN Bottom Reflection Gratings," Proc. SPIE., 7216, 72162Q72162Q-8 (2009).

${ }^{[16]}$ D. A. Steigerwald, J. C. Bhat, D. Collins, R. M. Fletcher, M. O. Holcomb, M. J. Ludowise, P. S. Martin, S. L. Rudaz, "Illumination with Solid State Lighting Technology," IEEE J. on Sel. Top. in Quantum Elec., 8, 310-320 (2002).

[17] K. Bao, X. Kang, B. Zhang, T. Dai, Y. Sun, Q. Fu, G. Lian, G. Xiong, G. Zhang, Y. Chen, "Improvement of light extraction from GaN-based thin-film light-emitting diodes by patterning undoped GaN using modified laser lift-off," Appl. Phys. Lett., 92, 141104 (2008).

[18] J. Goodman, "Introduction to Fourier Optics", Third Edition, Roberts \& Company Publishers, 2005.

[19] S. Kim, K. Lee, J. Kim, M. Kwon, S. Park, "Fabrication of photonic crystal structures on light emitting diodes by nanoimprint lithography," Nanotechnology, 18(5), 055306-055311 (2007).

[20] T. Wriedt, “A Review of Elastic Light Scattering Theories," Part. Part. Syst. Charact., 15, 67-74 (1998).

[21] S. Trieu and X. Jin, "Study of Top and Bottom Photonic Gratings on GaN LED with Error Grating Models," IEEE J. Quantum Electton., 46(10), 1456-1469 (2010). 